\title{
Prediction of Transcription Factor Families Using DNA Sequence Features
}

\author{
Ashish Anand ${ }^{1}$, Gary B. Fogel ${ }^{2}$, Ganesan Pugalenthi ${ }^{1}$, and P.N. Suganthan ${ }^{1}$ \\ ${ }^{1}$ School of Electrical and Electonic Engineering, Nanyang Technological University, \\ 50 Nanyang Avenue, 639798, Singapore \\ \{ashi0005, ganesan, epnsugan\}@ntu.edu.sg \\ ${ }^{2}$ Natural Selection, Inc. 9330 Scranton Road, Suite 150, San Diego, CA 92121 \\ gfogel@natural-selection.com
}

\begin{abstract}
Understanding the mechanisms of protein-DNA interaction is of critical importance in biology. Transcription factor (TF) binding to a specific DNA sequence depends on at least two factors: A protein-level DNA-binding domain and a nucleotide-level specific sequence serving as a TF binding site. TFs have been classified into families based on these factors. TFs within each family bind to specific nucleotide sequences in a very similar fashion. Identification of the TF family that might bind at a particular nucleotide sequence requires a machine learning approach. Here we considered two sets of features based on DNA sequences and their physicochemical properties and applied a one-versus-all SVM (OVA-SVM) with class-wise optimized features to identify TF family-specific features in DNA sequences. Using this approach, a mean prediction accuracy of $\sim 80 \%$ was achieved, which represents an improvement of $\sim 7 \%$ over previous approaches on the same data.
\end{abstract}

Keywords: Transcription factor family prediction, multi-class classification.

\section{Introduction}

Protein-DNA interactions play a central role in many cellular processes including transcription and translation. A key aspect of transcriptional regulation requires the binding of a class of proteins (called transcription factors (TFs)) to cis-acting DNA regulatory sequences (known as transcription factor binding sites (TFBS)). Understanding the mechanisms of these interactions and identifying associations between each TF and DNA regulatory elements are key challenges for experimental and computational biology.

TFBS are usually very short ( $\leq 12$ base pairs) [1] and some proteins are capable of binding to many TFBSs. Binding of a TF to the appropriate TFBS depends on two factors: A three-dimensional protein structure of the TF that presents an appropriate DNA-binding domain and the specific sequence of nucleotides recognized by TF. Though variability in TFBSs does exist, TFBSs share enough similarity such that they can be easily recognized in the nucleus by TF proteins. TFs can be classified into families based on these protein and DNA-binding characteristics and catalogs of 
TFBS and TF proteins can be found including JASPAR [2], Transfac [3]. Transfac uses the sequence similarities in TFBS as a basis of classification of TFs, whereas JASPAR uses the binding profiles (discussed below) to classify TFs into families.

Several approaches to identify TFBS and associate them with the binding TF exist. These include phylogenetic footprinting [4], position-specific scoring matrix (PSSM)based approaches [5], Gibbs sampling [6], and expectation-maximization [7]. Phylogenetic footprinting has been applied to genomic sequences to identify novel TFBSs [8]. Clustering the results of phylogenetic footprint analysis on sets of co-regulated genes can result in novel TFBS as well as the identification and annotation of previously identified TFBSs [9]. Approaches based on comparative genomics or phylogenetic footprinting require genomic sequences from several species and as a result are computationally intensive. In some cases there may not simply exists sufficient representation over the phylogenetic history to generate meaningful comparisons at the species level.

A PSSM or position-weight matrix (PWM) is used commonly as probabilistic representation of a TFBS. These matrices store frequencies of each nucleotide at each position of the binding site. Such models generally assume independence between nucleotides over all positions must use a fixed-length (typically arbitrary) TFBS, are unable to represent sequence properties such as sequence-dependent physicochemical properties [10].

These methods are used generally to identify TFBS in newly sequenced data and do not attempt to predict a putative TF for each identified binding site.

Recently, several approaches have been proposed to handle this problem. Narlikar and Hartemink [11] used sparse multinomial logistic regression (SMLR) [12], to predict TF family given a set of TFBSs. For a given set of DNA sequences, a set of nucleic-acid based sequence features were generated. These features were used to generate the model and predict the TF family.

Sandelin and Wasserman [2] used binding sites profiles to classify wellcharacterized TFs into "familial binding profiles." The database JASPAR was generated using such "familial binding profiles" with corresponding TFs and associated binding sites. First a collection of PSSM models for a TF were assembled and similarities between models were calculated. Finally, an assembly algorithm was used to compile all models into a single familial binding profile. Using this approach, TF binding sites were classified into 11 families. A brief description of the families examined in this study is given in the Materials and Methods section below. Prediction of TF-family for a given set of DNA binding sites can be accomplished using "familial profiles."

Tan et al. [13] utilized the information of comparative genomics to connect TFs with their corresponding TFBSs. Three mutually independent information methods were used to connect a DNA binding motif to a given TF. Comparative analysis of multiple genomes was used to generate two of these sources of information and the third was derived from similarities of TFBS interactions. For a given TF and DNA motif, the three types of information were combined to obtain the probability that such a pair was a true pair.

Narlikar and Hartemink [11] showed that the nucleic-acid based features of TFBS can be used to predict the families of corresponding binding TF. They demonstrated that the selected features are family-specific. Motivated by these results, we used 
sequence-based conformational and physicochemical features [10, 14] in addition to the features proposed by Narlikar and Hartemink [11] to develop models with an SVM-based classifier [15].

The results of this approach were compared directly to SMLR [11]. The addition of physicochemical features to the nucleic-acid based features led to significant improvement in predictive accuracy. The SVM-based classifier outperformed SMLR when only the nucleic-acid based features were used. Both SVM-based classifier and SMLR resulted in competitive predictive accuracies using additional set of physicochemical features.

\section{Materials and Methods}

\subsection{Datasets}

JASPAR is the largest, curated, and open-access collection of eukaryotic TFBS profile matrices [16]. TFBSs are classified in JASPAR into the 11 structural families shown in Table 1. As part of our experimental design, we only made use of those TFBS classes with 4 or more samples (see below). Given this requirement, two TFBS-families (bZIP-cEBP and TRP (MYB)) were removed (Table 1). The remaining 55 TFs from 9 TFBS-families were used for modelling. These families are briefly described below.

ETS Family: TFs belonging to this family contains a region of 85-90 AAs known as the erythroblast transformation specific (ETS) domain. This domain is quite rich in positively-charged and aromatic residues. The ETS domain binds to purine-rich segments of DNA [17].

bZIP-CREB Family: cAMP responsive element binding proteins (bZIP/CREB) are conserved, nuclear, bZIP-domain, dimeric transcription factors. TFs of this family

Table 1. TF families of JASPAR database. Abbreviations for some families are provided in square brackets.

\begin{tabular}{lcc}
\hline TF Family & Number of Samples & $\begin{array}{c}\text { Considered in this } \\
\text { Study }\end{array}$ \\
\hline ETS & 7 & Yes \\
bZIP-CREB & 4 & Yes \\
REL & 5 & Yes \\
Nuclear Receptor [NR] & 8 & Yes \\
Forkhead [Fkh] & 4 & Yes \\
bZIP-cEBP & 3 & No \\
bHLH (zip) & 9 & Yes \\
MADS & 5 & Yes \\
TRP (MYB) & 3 & No \\
Homeobox [Hbox] & 7 & Yes \\
HMG & 6 & Yes \\
\hline Total Samples & 61 & 55 \\
\hline
\end{tabular}


contact the DNA through a basic region generally found in the amino-terminus of the TF. They contain leucine zipper segments consisting of leucine or similar hydrophobic AA spaced roughly every 7 or 8 residues.

REL Family: The Rel homology domain is found mainly in eukaryotic TFs. TFs containing the domain do not use well-defined secondary structure for DNA-binding [18]. The domain is composed of two immunoglobulin-like beta barrel sub-domains which grips the DNA in major groove.

Nuclear Receptor: The DNA-binding domain of nuclear receptors is composed of two zinc finger motifs that differ in size, composition, and function. Each finger contains four cysteine residues coordinating one zinc ion. The zinc coordinating motif is characterized by two anti-parallel alpha-helices capped by loops at their aminoterminal ends. Normally TFs of this class function as homo- or heterodimers. Each monomer typically consists of ligand-binding, DNA-binding, and transcription regulatory domains.

Fork head: The fork head domain contains neither homeodomains nor zinc-finger characteristics of other TFs. It contains a distinct type of DNA binding region of around 100 AAs and binds B-DNA as monomer.

bHLH (zip): TFs of this family contain a tripartite DNA binding domain consisting of a basic region, a helix-loop-helix (HLH), and a leucine zipper. The domain mediates dimerization as a prerequisite for DNA-binding. The basic region dictates DNAbinding specificity. The leucine zipper consists of repeated leucine residues at every seventh position.

MADS: The MADS box is a highly conserved sequence motif found in a family of TFs. The conserved domain was recognized after the first four members of the family, MCM1, AGAMOUS, DEFICIENS, and serum response factor (SRF) and named after them by taking their initials. TFs belonging to this class function as dimers. The primary DNA-binding element is an anti-parallel coiled coil of two amphipathic $\alpha$ helices, one from each subunit. The MADS domain is a 56-residue motif consisting of a pair of anti-parallel coiled coil $\alpha$-helices packed against an anti-parallel, doublestranded, $\beta$-sheet.

Homeobox: The homeodomain binds through a helix-turn-helix (HTH) structure. HTH motifs are characterized by two $\alpha$-helices, joined by a short turn. Protein-DNA contacts are conserved, especially those made by positions R3, R5, I47, Q50, N51 and M54 [19]. This domain binds to DNA both as monomer and dimer. Some proteins are capable of both.

HMG: Proteins of this class comprise a region of homology with HMG proteins such as HMG1. Generally HMG domains bind DNA to non-sequence-specific manner. The domain exhibits an L-shaped configuration by 3 alpha helices. The $1^{\text {st }}$ and $2^{\text {nd }}$ helices contact DNA and the $3^{\text {rd }}$ helix is exposed to solvents.

\subsection{Feature Formulation}

Nucleic acid-based sequence features were used to represent each TF. Two main sets of features were defined: DNA features and DNA-Physico features (described in 
greater detail below). Features corresponding to DNA were calculated using only known binding sites or DNA motifs obtained from the JASPAR database. Flanking sequences were avoided given there is no general consensus on the notion of an "ideal" length for such flanking sequences in model development. Only the DNAbinding domain of TFs was used for feature calculation. For each TF, a list of binding DNA motifs or sites was obtained from the JASPAR database. Features corresponding to each DNA motif were calculated and average was taken to get single feature vector representing each TF. All features and their combinations are described in greater detail below.

1. DNA Features: We used the same set of features as discussed in [11]. These features included:

A) The frequency of subsequence features representing the counts of all subsequences of length 1 to 5 in each TFBS. Only the four nucleotides A, T, G, C were considered. The full 15-letter code was not considered as no consensus sequences in any form were taken as binding sites. 1,364 features were generated in this manner.

B) Ungapped palindrome features: Binary variables representing the presence or absence of palindrome subsequence of half-length $3,4,5$ or 6 spanning entire site and that of palindrome subsequence not spanning the whole length. Thus there were total 8 such binary features. It is important to mention here this set of features will be sparse. For example, the presence of subsequence of half-length 3 spanning entire site will make the rest 7 binary variables 0 .

C) Gapped palindrome features: The same as the above with one difference of possibility of gaps. Here gap indicates the insertion of some nonpalindrome nucleotides exactly in the middle of two palindrome halves. Similar to the previous case, total count of such features was 8.

D) Special features: Narlikar and Hartemink [11] identified 7 special sequence features from literature which are found to be over-represented in the binding sites of certain TF families. These seven features were $\mathrm{G}$. . G, G . . G . . G, [GC] . [GC] . [GC], AGGTCA I TGACCT, CA . . TG, TGA * * TCA, and TAAT I ATTA. Here ' ' means presence of any single nucleotide, ' $*$ ' means presence of at least one nucleotide, $[\mathrm{XY}]$ means presence of one of the letters $\mathrm{X}$ or $\mathrm{Y}$, and ' $\mathrm{XYZ}$ । $\mathrm{ABC}$ ' means presence of one of the strings ' $\mathrm{XYZ}$ ' or 'ABC'. The presence or absence of each of these features was used as additional features.

When concatenated, features 1A-D above resulted in a single feature vector of length 1,387 . The classifier model using solely this feature vector was referred to as the "DNA-model".

2. DNA-Physico Features: Conformational and physicochemical properties have been shown to affect the activity of cis-regulatory DNA elements [10, 14]. The mean values of 38 conformational and physicochemical properties of di-nucleotides were downloaded from the Property subdirectory of the Activity database [20]. For a given DNA site ' $S=s_{l}, s_{2}, \ldots, s_{i}, . . s_{L}$ ' of length $L$ a value representing each of the 38 features was calculated as follows: 


$$
X_{q}^{i}(S)=\frac{\sum_{j=1}^{L-1} P_{q}\left(s_{j}, s_{j+1}\right)}{L}
$$

where $P_{q}$ is the $q$ th property of dinucleotides $\left(s_{j}, s_{j+1}\right)$. There are only 38 such properties and when these were combined with the DNA feature set above, a total of 1,425 features resulted. The classifier model based on this feature set was referred to as the "DNA-Physico model."

\subsection{Model Description}

Background of SVM, OVA-SVM and SVM-RFE (OVA-RFE). Support vector machines (SVMs) [21] belong to the family of margin-based classifiers and can often achieve superior classification performance when compared to other classification algorithms across many domains. SVMs were originally designed to solve binary classification problems. Several algorithms have extended binary SVMs for application in multi-class problems [22-26]. One-versus-all (OVA) is one such simple and early extension of SVM for multi-class problems [27].

SVM-recursive feature selection (SVM-RFE) [28] was originally proposed for binary classification problems. The method of SVM-RFE begins with the set of all features and selectively eliminates one feature at a time. Features are scored and ranked on squared coefficients $w_{j}^{2}(j=1,2, \ldots, p)$ of weight vector $\mathbf{w}$. The feature with smallest $w_{j}^{2}$ is eliminated in each iterative step. The procedure is repeated until a predetermined number of features remain. This procedure can also be generalized to remove more than one feature per step [28]. SVM-RFE is also extended in OVA fashion by many researchers [29-31]. This extension is generally known as OVA-RFE.

Feature selection was performed in OVA-RFE fashion. Selected features were then used with corresponding OVA-SVM classifiers. Final class-prediction was made using probabilities scores obtained from OVA-SVMs. We have shown [15] that conversion of decision function values into probability scores increases predictive performance. Among the three methods of converting decision function values into probability scores that were evaluated previously [15]; Platt's approach [32] was determined to provide better or equivalent predictive accuracy over several data sets. Thus for the purpose of the current investigation, we used Platt's approach to convert the decision function values into probability scores.

\subsection{Experimental Design}

During pre-processing, for each feature type, redundant features with identical values over all samples were removed. The remaining features were normalized to $[-1,1]$. Table 2 lists number of features before and after pre-processing step.

Table 2. Feature Statistics

\begin{tabular}{lcc}
\hline Feature Type & Number of Features & \# Features After Pre-processing \\
\hline DNA & 1387 & 1305 \\
DNA-Physico & 1425 & 1343 \\
\hline
\end{tabular}


The performance of all models was assessed using $k$-fold external cross-validation (CV) following Ambroise and McLachlan [33] to provide an unbiased estimate of generalization error. CVs were performed 100 times to provide more reliable estimates of prediction accuracy. A linear kernel was used for the SVM and hence only one SVM parameter $(C)$ required tuning. For each model, a range of $C$ was evaluated $\left\{10^{-5}, 10^{-4}, 10^{-3}, 10^{-2}, 10^{-1}, 1\right\}$. The model and $C$ setting with best average CV (4-fold $\mathrm{CV}$ ) accuracy over all 100 runs was selected as the most appropriate setting of $C$. For "this purpose, all features were used and no feature selection was performed.

For feature selection, $3 / 4$ of the data were considered were used for the feature selection process and the best features were re-evaluated on the remaining $1 / 4$ of the data for performance. Average 4-fold accuracy was calculated. This procedure was repeated for 100 different stratified (i.e., the class-wise proportion in training set was kept the same as was in the whole set) partitions of 4-fold. Average CV accuracies over 100 runs were calculated to estimate the prediction accuracy of models. We started with all features and successively eliminated $1 \%$ of remaining features in each iteration of OVA-RFE until a minimum of 10 features were left.

For fair comparison to SMLR [11], we used the same normalized data as was used in OVA-experiments. Different values of the parameter $\lambda$ were tried and the one giving best average 4-fold CV accuracy over 100 runs was reported. We used SMLR software [12] available from the http://www.cs.duke.edu/ amink/software/smlr/.

\section{Results and Discussion}

\subsection{Comparison between SMLR and Class-Wise Optimized OVA-SVM}

Table 3 lists prediction accuracies obtained by models using different feature-types. OVA-SVM approach performed significantly better than SMLR using only DNA features $\left(t\right.$-test $p$-value $\left.=1.65 \times 10^{-43}\right)$, however there was no significant difference found between the two approaches when DNA-Physico features was used. The mean predictive accuracy of SMLR approach improved significantly (from 74.05\% $\pm 3.34 \%$ to $81.91 \% \pm 2.69 \%$; t-test $\mathrm{p}$-value $=1.68 \times 10^{-44}$ ) with use of DNA-Physico features.

Table 3. Performance comparison of SMLR and Class-wise optimized OVA-SVM

\begin{tabular}{llll}
\hline Type of Features & $\begin{array}{l}\text { OVA-SVM } \\
\text { (All-Feats) }\end{array}$ & $\begin{array}{l}\text { OVA-SVM } \\
\text { (Feat-Selection) }\end{array}$ & SMLR \\
\hline DNA & $81.44 \pm 3.04$ & $\begin{array}{l}\mathbf{8 1 . 8 6} \pm \mathbf{2 . 7 7}(773) \\
80.88 \pm 2.98(200)\end{array}$ & $\begin{array}{l}74.05 \pm 3.34 \\
(\lambda=0.001)\end{array}$ \\
& & $80.06 \pm 3.07(35)$ & \\
& & & \\
DNA-Physico & $81.99 \pm 3.07$ & $\mathbf{8 2 . 5 6} \pm \mathbf{2 . 8 2}(471)$ & $81.91 \pm 2.69$ \\
& & $81.73 \pm 2.64(60)$ & $(\lambda=1.0 \mathrm{E}-5)$ \\
& & $80.09 \pm 2.88(30)$ & \\
\hline
\end{tabular}




\subsection{DNA-Model and DNA-Physico-Model Features}

The addition of physicochemical properties improved mean prediction accuracy, though not significantly for the OVA-SVM approach (t-test p-value $=0.20$ ). Figure 1 compares the average error obtained by models using different number of DNA and DNA-Physico features. Feature selection did not lead to any significant improvement in mean predictive accuracy however; more parsimonious models could be obtained using far fewer features with similar mean accuracy. For example, using only 35 DNA features, a mean prediction accuracy of $80.06 \% \pm 3.07 \%$ was generated. Similarly, using 30 DNA-Physico features generated a mean prediction accuracy of $80.09 \% \pm 2.88 \%$. To compare the best predictive accuracy obtained by the two models irrespective of the number of features used, the model using DNA features only obtained best accuracy of $81.86( \pm 2.77)$ by using 773 features per class and the best accuracy of $82.56( \pm 2.82)$ was obtained by the model using DNA-Physico features with 471 features per class (Table 3 ).

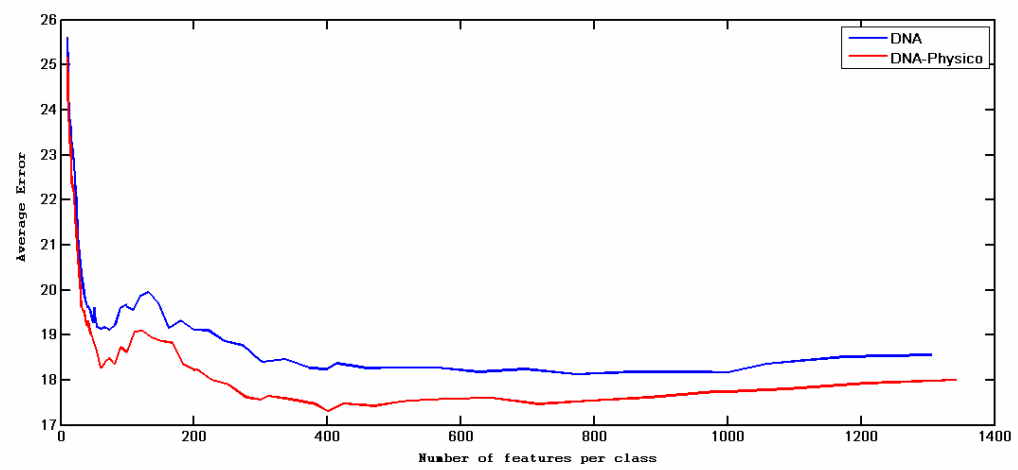

Fig. 1. Comparison between DNA and DNA-Physico Models

These results show that the model using DNA-Physico features was always able to provide slightly improved predictive accuracies than model using DNA features only. This suggests that the conformational and physicochemical features might influence the prediction of some of the TF families. We reviewed only the features which were selected more than $50 \%$ of the time by DNA-Physico models when using 30 features per TF family and separated these into conformational or physicochemical features (Table 4) to check our hypothesis. DNA-Physico features appeared to be important for the bHLH-ZIP family. We compared the prediction accuracy obtained by the two models for bHLH-ZIP family. The DNA-Physico model obtained an accuracy of $95.78 \% \pm$ $6.27 \%$ whereas the DNA model obtained an accuracy of $90.56 \% \pm 6.58 \%$. The statistical significance of this difference was evaluated using a proportion test but no statistical significance was observed ( $\mathrm{p}$-value $=0.49$ ) but reviewing the results as number of correct predictions made in each partitions shows a significant difference between the two models. The DNA-model predicts all 9 samples from the bHLH-ZIP family correctly only 26 times out of the 100 runs whereas the DNA-Physico model corrects all 9 samples of this family 66 times, for a difference of 40 out of 100 runs (Table 5). 
Table 4. Class-wise statistics of different feature types: Features with frequency more than 200 were considered only. This statistics is obtained from the model using DNA-Physico Features with 30 features per class.

\begin{tabular}{lccccccccc}
\hline $\begin{array}{l}\text { TF- } \\
\text { Family }\end{array}$ & ETS & $\begin{array}{l}\text { bZIP- } \\
\text { CREB }\end{array}$ & REL & NR & Fkh & $\begin{array}{l}\text { bHLH } \\
\text { (zip) }\end{array}$ & MADS & Hbox & HMG \\
\hline $\begin{array}{l}\text { \#DNA } \\
\text { Features }\end{array}$ & 28 & 22 & 31 & 26 & 27 & 19 & 27 & 23 & 19 \\
$\begin{array}{l}\text { \#Physico } \\
\text { Features }\end{array}$ & 2 & 0 & 0 & 0 & 0 & 5 & 0 & 2 & 0 \\
\hline
\end{tabular}

Table 5. Number of true classifications for bHLH-ZIP family by the two models in 100 partitions. Total number of samples in bHLH-ZIP was 9. Numbers in bracket in the first column indicates number of features used by OVA-SVM classifier.

\begin{tabular}{lccc}
\hline \# True Classifications & $\mathbf{7}$ & $\mathbf{8}$ & $\mathbf{9}$ \\
\hline DNA-model (35) & 11 & 63 & 26 \\
DNA-Physico-model (30) & 4 & 30 & 66 \\
\hline
\end{tabular}

\section{Conclusion}

In this paper, features based on the TFBS sequences and their physico-chemical properties were used to build an OVA-SVM based multi-class classifier to predict the family of an associated binding TF protein. A detailed study was conducted to investigate the importance of different feature types for this decision. The performance of OVA-SVM based multi-class classifier and SMLR were compared and a significant improvement was found in the performance of SMLR when additional physicochemical features were added to the nucleic-acid based features. While OVA-SVM outperformed SMLR based on only DNA-features, performance of the methods were competitive when DNA-Physico features were used.

Acknowledgments. Authors acknowledge the financial support offered by the A*Star (Agency for Science, Technology, and Research, Singapore) under the grant \#052 101 0020 .

\section{References}

1. Fogel, G.B., Weekes, D.G., Varga, G., Dow, E.R., Craven, A.M., Harlow, H.B., Su, E.W., Onyia, E., Su, C.: A Statistical Analysis of the TRANSFAC database. Biosystems 81(2), 137-154 (2005)

2. Sandelin, A., Wasserman, W.W.: Constrained Binding Site Diversity within Families of Transcription Factors Enhances Pattern Discovery Bioinformatics. J. Mol. Biol. 338, 207215 (2004) 
3. Matys, V., et al.: TRANSFAC: Transcriptional Regulation, from Patterns to Profiles. Nucleic Acids Res. 31, 374-378 (2003)

4. Blanchette, M., Tompa, M.: Discovery of Regulatory Elements by a Computational Method for Phylogenetic Footprinting. Genome Research 12(5), 739-748 (2002)

5. Stormo, G.: DNA Binding Sites: Representation and Discovery. Bioinformatics 16, 16-23 (2000)

6. Lawrence, C.E., Altschul, S.F., Boguski, M.S., Liu, J.S., Neuwald, A.F., Wootton, J.C.: Detecting Subtle Sequence Signals: A Gibbs Sampling Strategy for Multiple Alignment. Science, 208-214 (1993)

7. Bailey, T.L., Elkan, C.: Fitting a Mixture Model by Expectation Maximization to Discover Motifs in Biopolymers. In: Second International Conference on Intelligent Systems for Molecular Biology, pp. 28-36. AAAI Press, Menlo Park (1994)

8. McCue, L.A., Thompson, W., Carmack, C.S., Lawrence, C.E.: Factors Influencing the Identification of Transcription Factor Binding Sites by Cross-Species Comparis. Genome Res. 12, 1523-1532 (2002)

9. Qin, Z.S., McCue, L.A., Thompson, W., Mayerhofer, L., Lawrence, C.E., Liu, J.S.: Identification of Co-reguated Genes through Bayesian Clustering of Predicted Regulatory Binding Sites. Nature Biotechnology 21, 435-443 (2003)

10. Ponomarenko, J., Ponomarenko, M., Frolov, A., Vorobyev, D., Overton, G., Kolchanov, N.: Conformational and Physicochemical DNA Features Specific for Transcription Factor Binding Sites. Bioinformatics 15, 654-668 (1999)

11. Narlikar, L., Hartemink, A.J.: Sequence Features of DNA Binding Sites Reveal Structural Class of Associated Transcription Factor. Bioinformatics 22(2), 157-163 (2006)

12. Krishnapuram, B., Figueiredo, M., Carin, L., Hartemink, A.: Sparse Multinomial Logistic Regression: Fast Algorithms and Generalized Bounds. IEEE Transactions on Pattern Analysis and Machine Intelligence 27, 957-968 (2005)

13. Tan, K., McCue, L.A., Stormo, G.D.: Making Connections between Novel Transcription Factors and their DNA Motifs. Genome Res. 15, 312-320 (2005)

14. Anand, A., Fogel, G., Tang, E.K., Suganthan, P.N.: Feature Selection Approach for Quantitative Prediction of Transcriptional Activities. In: IEEE Symposium on Computational Intelligence in Bioinformatics and Computational Biology, Toronto, Canada, pp. 57-62 (2006)

15. Anand, A., Pugalenthi, G., Suganthan, P.N.: Predicting Protein Structural Class by SVM with Class-wise Optimized Features and Decision Probabilities. Journal of Theoretical Biology (2008), doi:10.1016/j.jtbi.2008.02.031

16. Vlieghe, D., Sandelin, A., De Bleser, P.J., Vleminckx, K., Wasserman, W.W., Roy, F., Lenhard, B.: A New Generation of JASPAR, the Open-Access Repository for Transcription Factor Binding Site Profiles. Nucleic Acids Research 34, 95-97 (2006)

17. Karim, F.D., et al.: The ETS-domain: a new DNA-binding motif that recognizes a purinerich core DNA sequence. Genes Dev. 4, 1451-1453 (1990)

18. Luscombe, N.M., Austin, S.E., Berman, H.M., Thornton, J.M.: An overview of the structures of protein-DNA complexes. Genome Biology. 1(1), reviews001.1—001.10 (2000)

19. Svingen, T., Tonnisen, K.F.: Hox Transcription Factors and their Elusive Mammalian Gene Targets. Heredity 97, 88-96 (2006)

20. Ponomarenko, J.V., Furman, D.P., Kolchanov, N.A., Sarai, A.: Activity: A database on DNA/RNA sites activity adapted to apply sequence-activity relationships from one system to another. Nucleic Acids Research 29(1), 284-287 (2001)

21. Vapnik, V.: Statistical learning theory. Wiley-Interscience, New York (1998) 
22. Kreßel, U.H.-G.: Pairwise classification and support vector machines. In: Scholkopf, B., Burges, C.J.C., Smola, A.J. (eds.) Advances in kernel methods: Support vector learning, pp. 255-268. MIT press, Cambridge (1999)

23. Weston, J., Watkins, C.: Support vector machines for multiclass pattern recognition. In: Proc 7th European symposium on artificial neural networks (1999)

24. Crammer, K., Singer, Y.: On the algorithmic implementation of multiclass kernel-based vector machines. Journal of machine learning research 2, 265-292 (2001)

25. Hsu, C.-W., Lin, C.-J.: A comparison of methods for multi-class support vector machines. IEEE Trans. Neural Networks 13, 415-425 (2002)

26. Lee, Y., et al.: Multicategory support vector machines: theory and application to the classification of microarray data and satellite radiance data. J. Am. Stat. Assoc. 99, 67-81 (2004)

27. Bottou, L. et al.: Comparison of classifier methods: A case study in handwriting digit recognition. In: Proc. Int. Conf. Pattern Recognition. pp. 77-87 (1994)

28. Guyon, I., Weston, J., Barnhill, S., Vapnik, V.: Gene selection for cancer classification using support vector machines. Machine Learning 46, 389-422 (2002)

29. Chai, H., Domeniconi, C.: An evaluation of gene selection methods for multi-class microarray data classification. In: Scheffer, T. (ed.) Proceedings of the Second European Workshop on Data Mining and Text Mining in Bioinformatics, pp. 3-10 (2004)

30. Ramaswamy, S., Tamayo, P., Rifkin, R., Mukherjee, S., Yeang, C.H., Angelo, M., Ladd, C., Reich, M., Latulippe, E., Mesirov, J.P., et al.: Multiclass cancer diagnosis using tumor gene expression signatures. Proc. Natl. Acad. Sci. USA 98, 15149-15154 (2001)

31. Rifkin, R., Mukherjee, S., Tamayo, P., Ramaswamy, S., Yeang, C.-H., Angelo, M., Reich, M., Poggio, T., Lander, E.S., Golub, T.R., Mesirov, J.P.: An analytical method for multiclass molecular cancer classification. Siam Review 45(4), 706-723 (2003)

32. Platt, J.: Probabilistic outputs for support vector machines and comparison to regularized likelihood methods. In: Smola, A.J., Bartlett, P.L., Scholkopf, B., Schuumans, D. (eds.) Advances in Large Margin Classifiers, pp. 61-74. MIT Press, Cambridge (2000)

33. Ambroise, C., McLachlan, G.J.: Selection bias in gene extraction on the basis of microarray gene-expression data. Pro. Nat. Acad. Sci. 99, 6562-6566 (2002) 